\section{RMD Open}

Rheumatic \&

Musculoskeletal Diseases

\title{
Diabetes mellitus is not a risk factor for osteoarthritis
}

\author{
Andrew Khor (D) , ${ }^{1}$ Cheryl-Ann Ma, ${ }^{2}$ Cassandra Hong, ${ }^{1,2}$ Laura Li-Yao Hui, ${ }^{1}$ \\ Ying Ying Leung (D) 1,2
}

To cite: Khor A, Ma C-A, Hong $C$, et al. Diabetes mellitus is not a risk factor for osteoarthritis. RMD Open 2020;6:e001030. doi:10.1136/ rmdopen-2019-001030

- Additional material is published online only. To view please visit the journal online (http://dx.doi.org/10.1136/ rmdopen-2019-001030).

Received 11 June 2019 Revised 18 December 2019 Accepted 21 December 2019

\section{ABSTRACT}

Background Association between diabetes mellitus (DM) and risk of osteoarthritis (OA) can be confounded by body mass index (BMI), a strong risk factor for both conditions. We evaluate the association between DM or hyperglycaemia with $0 \mathrm{~A}$ using systematic review and meta-analysis.

Methods We searched PubMed and Web of Science databases in English for studies that gave information on the association between DM and OA. Two metaanalysis models were conducted to address: (1) risk of DM comparing subjects with and without $O A$ and (2) risk of $O A$ comparing subjects with and without DM. As far as available, risk estimates that adjusted for BMI were used.

Results 31 studies with a pooled population size of 295100 subjects were reviewed. 16 and 15 studies reported positive associations and null/ negative associations between DM and $0 \mathrm{~A}$. $68.8 \%$ of positive studies had adjusted for BMI, compared with $93.3 \%$ of null/negative studies. In meta-analysis model 1 , there was an increase prevalence of DM in subjects with $\mathrm{OA}$ compared with those without (OR 1.56, 95\% Cl 1.28 to 1.89). In meta-analysis model 2 , there was no increased risk of $\mathrm{OA}(\mathrm{OR} 1.14,95 \% \mathrm{Cl} 0.98$ to 1.33$)$ in subjects with DM compared with those without, regardless of gender and $\mathrm{OA}$ sites. Comparing subjects with DM to those without, an increased risk of OA was noted in crosssectional studies, but not in case-control and prospective cohort studies.

Conclusions This meta-analysis does not support DM as an independent risk factor for $\mathrm{OA}$. BMI was probably the most important confounding factor.

\section{INTRODUCTION}

\section{(c) Author(s) (or their} employer(s)) 2020. Re-use permitted under CC BY-NC. No commercial re-use. See rights and permissions. Published by BMJ.

${ }^{1}$ Rheumatology and Immunology, Singapore General Hospital, Singapore, Singapore ${ }^{2}$ Duke-NUS Medical School, Singapore, Singapore

\section{Correspondence to} Dr Ying Ying Leung; katyccc@hotmail.com
Osteoarthritis (OA) is one of the leading causes of disability globally with increasing burden, ${ }^{1}$ driven mainly by increasing age and obesity. ${ }^{2}$ Our group has previously demonstrated that increasing body mass index (BMI) is a very strong risk factor for OA, even in nonobese ranges. ${ }^{3}$ In addition to the mechanical stress caused by weight that contributes to the development of OA, evidence has suggested that metabolic mediators of obesity including diabetes mellitus (DM) or hyperglycaemia may play a role. However, whether DM or

\section{Key messages}

What is already known about this subject?

- Obesity is a strong risk factor for development of osteoarthritis (OA).

- The association between diabetes mellitus (DM) or hyperglycemia and $\mathrm{OA}$ is less clear.

- There is conflicting results regarding the association between $\mathrm{OA}$ and $\mathrm{DM}$ from existing studies.

What dose this study add?

- This study is a updated and comprehensive systemic review and the first meta-analysis to refute an independent association between $\mathrm{OA}$ and $\mathrm{DM}$.

- BMI was the most important confounding factor.

How might this impact on clinical practice?

- Future research should account for the effects of $\mathrm{BMI}$ as it is a major confounding factor in the development of $\mathrm{OA}$.

hyperglycaemia is a causative factor for $\mathrm{OA}$ independent from obesity remains controversial.

Obesity is well known to be a strong risk factor for DM or hyperglycaemia. ${ }^{4}$ Even in experimental animal studies, it is difficult to separate the biomechanical effects of obesity from the effects of diabetes. ${ }^{5}$ In studies conducted among Caucasian populations that have examined the association between DM or hyperglycemia and knee OA, subjects with DM or hyperglycaemia had much higher BMI compared with those without, ${ }^{6-8}$ raising the possibility of residual confounding effect of BMI despite statistical adjustments. On the other hand, a large prospective cohort study conducted in a lean population with comparable BMI between comparison groups has demonstrated a negative association between DM and knee OA. ${ }^{9}$

Recent systematic reviews have reported conflicting results. Both Louati et al and Williams et al reported a positive association between OA and DM. ${ }^{10} 11$ However, the former meta-analysis pooled crude odd 
ratios (ORs from studies that did not adjust for BMI. Although the latter meta-analysis reported a positive association between $\mathrm{OA}$ and $\mathrm{DM}$ that was maintained after adjustment of BMI, this meta-analysis involved a much fewer number of studies and did not include several more recent large studies which showed negative results. ${ }^{912} 13$ More recently, Dawson et al reported that there was little evidence to suggest impaired glucose metabolism as a risk factor for OA. ${ }^{14}$ However, it was a qualitative analysis which did not include a meta-analysis.

Given the close correlation between obesity and DM or hyperglycaemia in epidemiological studies, it is essential to acknowledge the confounding effect of BMI in the association between DM and risk of OA. There is therefore a need to clarify the association between DM and $\mathrm{OA}$ as it has implications on the pathogenesis of $\mathrm{OA}$ as well as the clinical management of both diseases. Hence, we conducted a systematic literature review on the association between DM or hyperglycaemia and risk of OA, with attention to how obesity has been adjusted as a confounding factor.

\section{METHODS}

\section{Literature search and selection of articles}

We performed a database search through PubMed and Web of Science for relevant original studies published in full text and in English up to 11 May 2017 that gave information on the association between DM or hyperglycaemia and the risk of onset or progression of OA. We focused on studies in human. The search terms for PubMed were: ("diabetes mellitus, type 2" $[\mathrm{MeSH}]$ or "diabetes mellitus, type 1" [MeSH] or "diabetes complications" $[\mathrm{MeSH}]$ or "metabolic syndrome X" $[\mathrm{MeSH}]$ or ("blood glucose" [MeSH] or "blood glucose" [All Fields])) and "osteoarthritis" [MeSH] and ("humans" $[\mathrm{MeSH}]$ and (English[lang])). The search terms for Web of Science were $(\mathrm{TS}=($ diabetes mellitus $)$ and $\mathrm{TS}=($ osteoarthritis) and TS=(human) $)$ and LANGUAGE: (English). An updated search from 11 may 2017 to 22 Jan 2018 was performed using PubMed using the same search terms. Two researchers (CM and YYL) reviewed the titles, abstracts and full-text articles (when appropriate) and selected eligible articles by consensus. A third researcher (AK) was involved in consensus meeting for selection of articles in cases of disagreement.

\section{Data extraction and quality assessment}

Four independent researchers worked in teams of two (YYL/AK, YYL/CH, YYL/LH) to extract data independently onto a standardised protocol, followed by consensus of the data. In cases where there was disagreement between the two researchers, a third researcher reviewed the data to achieve consensus. The data extraction protocol consisted of the following: study design (cross-sectional, case-control or prospective cohort) and population (community, registry or hospital), case definition for OA, site of OA, BMI or number of subjects with obesity, population size and the outcome (relative risk, OR or HR or conclusion).

Quality assessment for the selected studies was performed using Newcastle-Ottawa Scale (NOS) for case-control study or prospective cohort study where applicable. ${ }^{15}$ Studies were assessed according to three domains: selection, comparability and outcome for prospective cohort studies; and selection, comparability and exposure for case-control studies. Stars (*) were awarded for each of the categories, to a maximum of four stars for selection, two stars for comparability and three stars for outcome/exposure. For comparability, BMI and age were chosen as the main factors for consideration. According to NOS, one star is awarded for comparability if the study controls for the most important factor (BMI) and another star for the second most important factor (age), with a maximum of 2 stars were awarded for compatibility for each study. In this study, we specifically evaluated the comparability of BMI between DM with non-DM, and OA with non-OA for each study in a more stringent manner. A star for BMI compatibility was awarded to a study only if the difference in BMI between the comparison groups was less than $1 \mathrm{~kg} / \mathrm{m}^{2}$. For study with baseline BMI between comparison groups exceeding $1 \mathrm{~kg} / \mathrm{m}^{2}$, but have adjusted BMI in the statistical models, a half star (denoted by $(\varnothing)$ ) was awarded.

\section{Qualitative appraisal of studies}

We summarised studies in table formats separately for positive and null/negative studies. We presented in tables the study designs, sample sizes, difference in baseline BMI comparing participants with or without OA and participants with or without DM whichever were available. We made descriptive comparison between positive and negative studies for these baseline characteristics.

\section{Meta-analysis}

We performed comparative analysis for OR and 95\% confidence intervals (CIs) in assessing the association between DM or hyperglycaemia and OA. We performed the meta-analysis with a random effect model using Comprehensive Meta-analysis V.3. Data for each study were entered as sample size/events, ORs/CIs for crosssectional/case-control studies or hazard ratios (HRs)/ CIs for prospective cohort studies. These were computed and summarised as standardised $\mathrm{OR}$ and variance. We expect high heterogeneity of these epidemiology studies that have different study designs, definition of cases and were conducted and in multiple centres and countries with multiple ethnicities and thus cannot be assumed to share a common effect. Rather, the summary effect was an estimate of the mean of a distribution of true effect. ${ }^{16}$ Therefore, the random effect model was more appropriate and was used throughout.

Two models were conducted to address: (1) the risk of DM or hyperglycaemia comparing subjects with and 
without OA and (2) the risk of OA comparing subjects with and without DM. As far as data are available, we used the best adjusted model from each study that accounted for age, BMI and other covariates in the meta-analyses. For studies that have multiple subgroups comparing to the same non-case (controls), such as prevalence or progression of OA compared with controls, prevalence of OA was chosen in the main model. Similarly, if multiple sites of OA sites were compared with controls, one site of OA (knee as first choice) was chosen. For studies that had stratified analysis for subgroups (such as gender and age), data were presented as separate study data point in the meta-analysis.

For model 2, we performed additional sensitivity analyses with comparability of BMI in studies, first limited to studies that have adjusted for BMI in the statistical models and second limited to in studies that had comparable BMI between subjects with DM compared with without DM. Limiting to studies with comparable BMI between groups at baseline may allow the possible biometabolic effect of DM on risk of OA to be revealed more independently from the biomechanical effect of BMI. In addition, we conducted subgroup analyses to evaluate the effects of study design, gender and sites of OA on effect of risk estimates.

We tested publication bias by visual assessment using funnel plots and quantitatively using Egger's test $\left(p>0.05=\right.$ no publication bias). ${ }^{17}$ We also simulated missing studies using the 'Trim and Fill' method. ${ }^{18}$ The imputed studies were plotted with the observed studies in the funnel plot and the risk estimates were re-evaluated to give insight of possible change when the models were free of publication bias.

\section{RESULTS}

\section{Literature search results}

Our literature search through January 2018 identified a total of 337 articles. After removal of duplicates, 270 unique articles remained. Majority of these were excluded for the following reasons: irrelevant (217 articles), commentaries or reviews (35 articles), case reports (three articles) and meta-analysis (one article). An updated search added six more articles while 19 other articles were identified by cross reference checking. In total, 39 relevant articles were retrieved for full text review. Of these, two were excluded for lacking a control group without $\mathrm{OA}$, three were excluded as DM was evaluated as part of metabolic syndrome without separate data available for DM or hyperglycaemia and three were excluded for duplicated publications from same databases. The final review included 31 studies comprising 8 prospective cohort, 11 cross-sectional and 12 case-control studies (figure 1), representing a total study population of 295100 subjects.

\section{Study characteristics}

Of the 31 studies, 16 reported positive associations between DM or hyperglycaemia and OA (table 1), while 15 showed null/negative associations (table 2 ).

\section{Positive studies}

Studies that showed positive associations represented a pooled study population size of 108258 . Only three of these studies were prospective cohorts (table 1), representing 2782 subjects. As majority of the positive studies had small sample size, the results were mainly driven by one large cross-sectional study with a sample size of $81634,{ }^{19}$ for which BMI was not adjusted in the model. Overall, 11 out of 16 studies $(68.8 \%)$ had at least some form of adjustment for BMI. Two studies ${ }^{20}{ }^{21}$ (12.5\%) had comparable BMI between subjects with and without DM. Nine studies adjusted for BMI with statistical modelling. ${ }^{.822-28}$

\section{Null/negative studies}

The 15 studies that reported null or negative association between DM or hyperglycaemia and OA, represented a total population size of 186842 . There was a higher number of prospective cohort studies (five studies), ${ }^{99-32}$ in which exposure and covariate factors prior to the outcome were minimised. These 5 prospective cohort studies involved much higher number of 83857 subjects compared with the 2782 subjects involved in the positive studies with prospective cohort design (table 2). Within these five prospective cohort studies, four reported negative associations between DM and OA. ${ }^{9} 121333$

These null/negative studies generally had larger sample sizes than the positive studies. Fourteen out of 15 null/negative studies $(93.3 \%)$ had adjusted for BMI in the final models, while 5 studies $(33.3 \%)$ had comparable BMI between subjects with DM and those without. $^{93133-35}$

\section{Meta-analysis Model 1: Risk of DM comparing subjects with $O A$ to those without $O A$}

Eleven studies were analysed in the meta-analysis to assess risk of DM in subjects with OA compared with those without OA. The pooled population size was 209610. There was increased risk of DM comparing subjects with $\mathrm{OA}$ to those without (OR 1.56, 95\% CI 1.28 to 1.89 , heterogeneity $\mathrm{I}^{2}=94.2 \%$ ) (figure 2). Apart from one study, ${ }^{31}$ all studies did not adjust for body weight, obesity or BMI in the models. This pooled OR represented a higher crude prevalence of DM among subjects with OA compared with those without. Funnel plot (online supplementary figure 1) revealed no publication bias (Egger's test $\mathrm{p}=1.50$ ).

\section{Meta-analysis Model 2: Risk of OA comparing subjects with DM to those without DM}

Twenty-two studies were analysed in the meta-analysis to assess risk of OA in subjects with DM or hyperglycaemia compared with those without. Among the pooled 


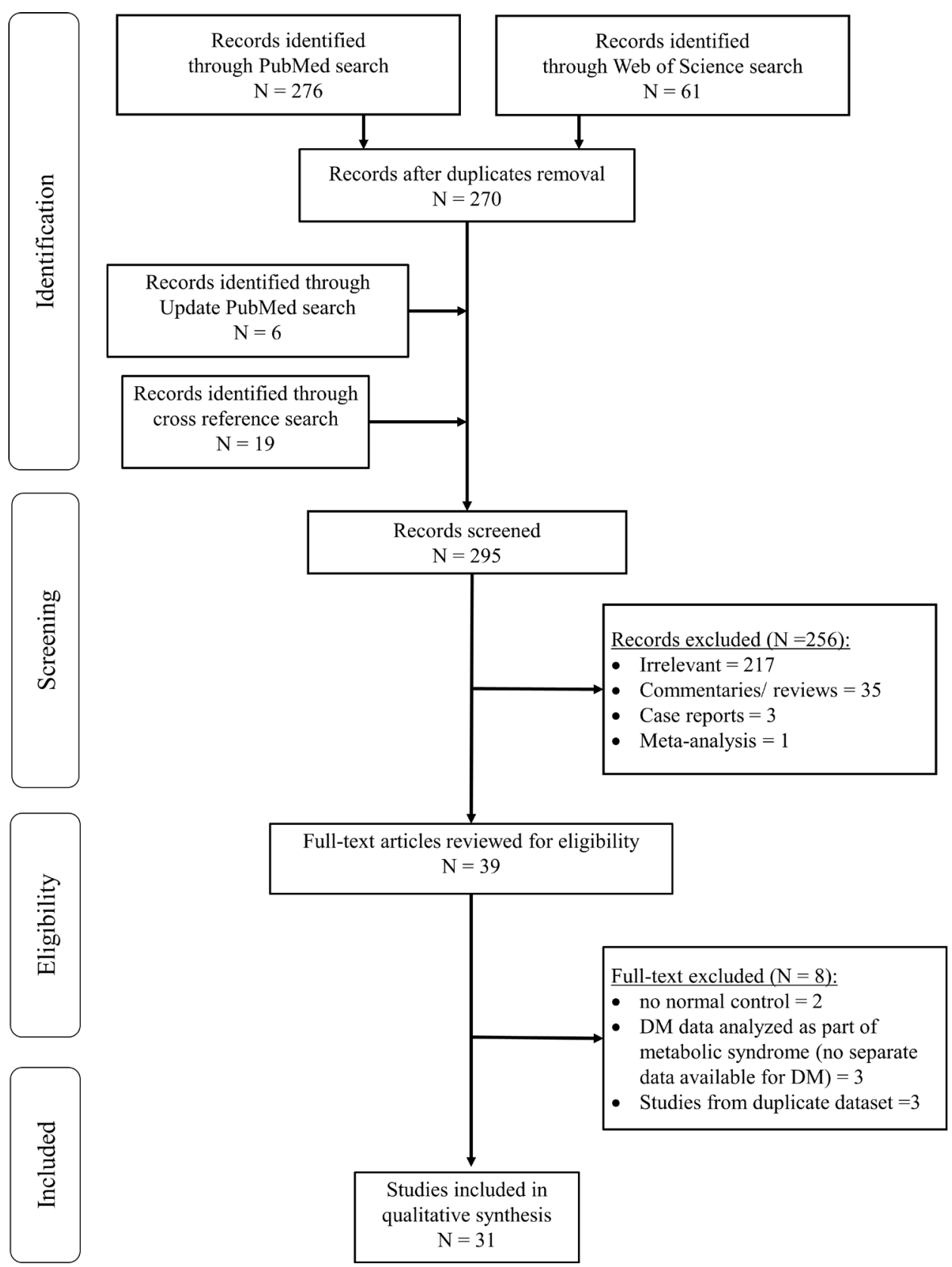

Figure 1 Flowchart for selection of articles. DM, diabetes mellitus.

population size of 200686 subjects, there was no significant association between DM and OA, (OR 1.14, 95\% CI 0.98 to 1.33 , heterogeneity $\mathrm{I}^{2}=74.2$ ) (figure 3 ). Two studies have not adjusted BMI in their statistical models, one of which did not provide detail on baseline BMI between DM and non-DM subjects, ${ }^{36}$ while another had comparable BMI between DM and non-DM subjects at baseline ${ }^{33}$ For the other 20 studies, the best adjusted OR that included BMI from studies were used. This model showed no significant increase risk of OA comparing subjects with DM and those without, when other confounding factors including BMI were accounted for. Funnel plot was asymmetrical with missing studies with negative associations (online supplementary figure 1), indicating possible publication bias (Egger's test $\mathrm{p}=0.02$ ).
The re-evaluated risk estimate was 1.06 (95\% CI 0.91 to 1.24).

In the sensitivity analyses that limit to studies with BMI adjustment in statistical models (20 studies), and studies with comparable baseline BMI between subjects with or without DM (seven studies), ${ }^{9} 203133-3537$ no significant associations were noted between $\mathrm{OA}$ and DM (OR=0.94, 95\% CI 0.68 to 1.29 ) (table 3). Publication bias with missing negative studies was revealed in model with adjusted BMI, but not in model with comparable baseline BMI (online supplementary figure 6 , table 3 ).

In the subgroup analysis stratified by study design, increased risk of OA was noted comparing DM with non-DM subjects in cross-sectional studies (OR 1.29, $95 \%$ CI 1.10 to 1.51 ), but not for case-control (OR 0.82, 


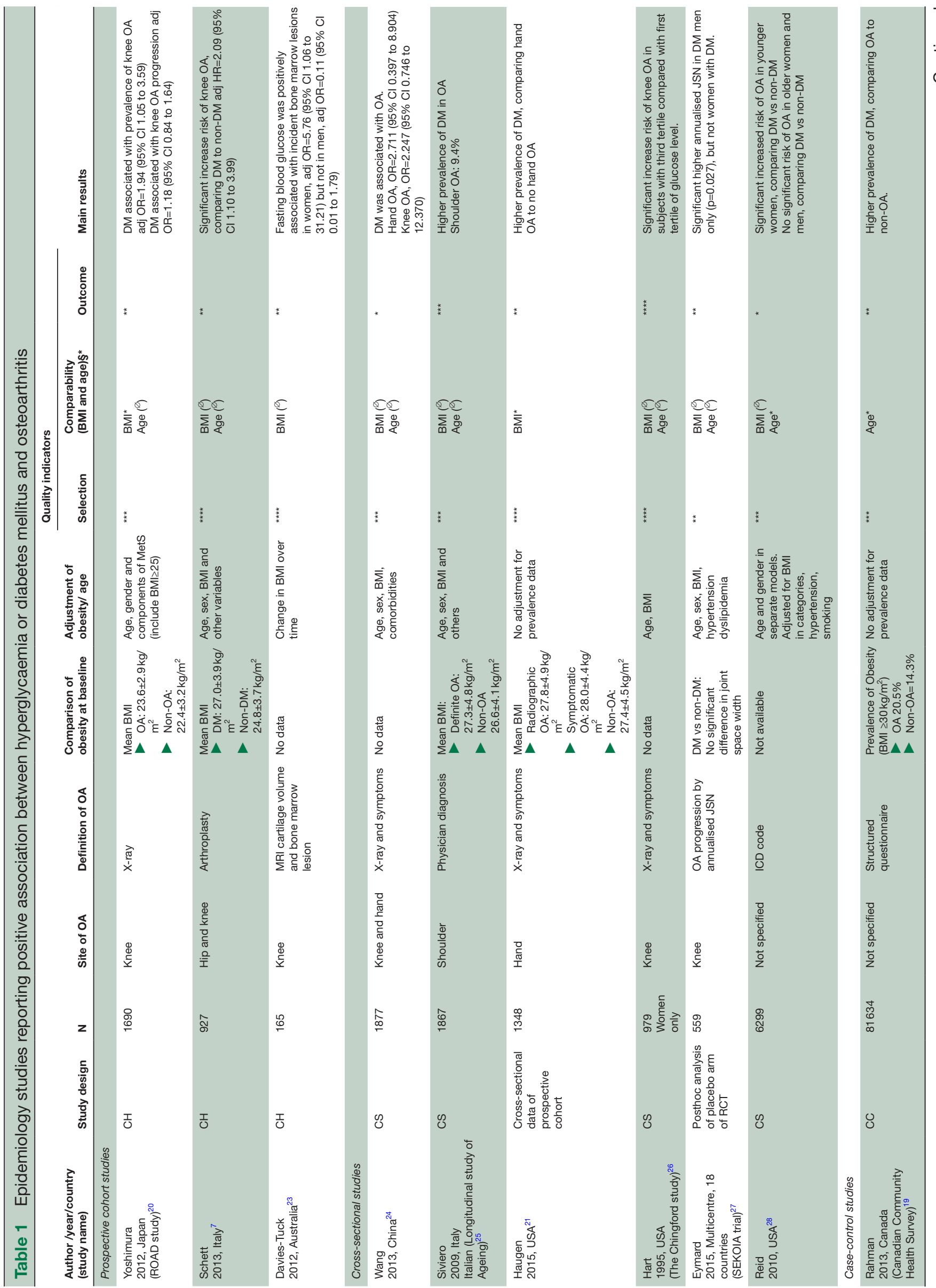




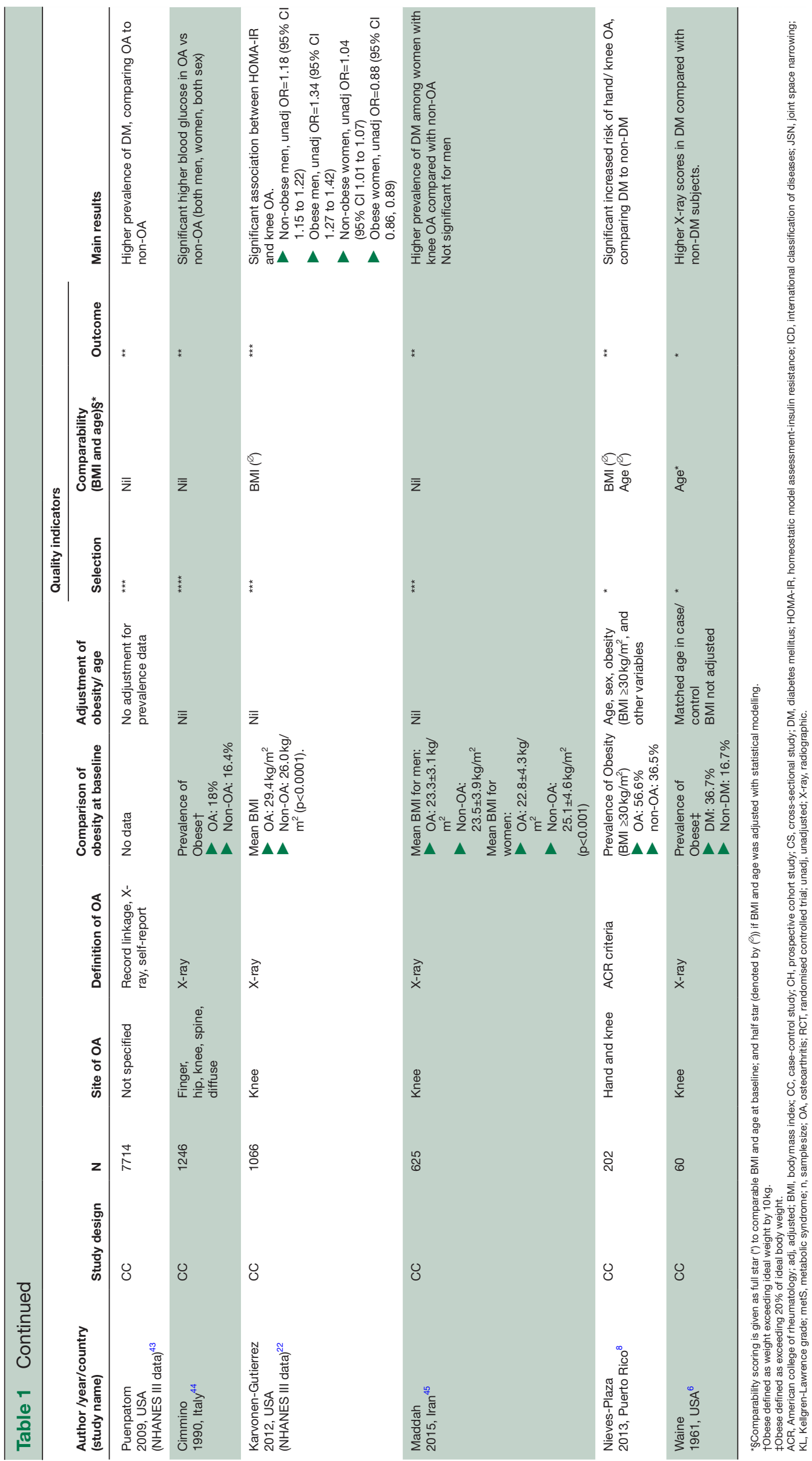




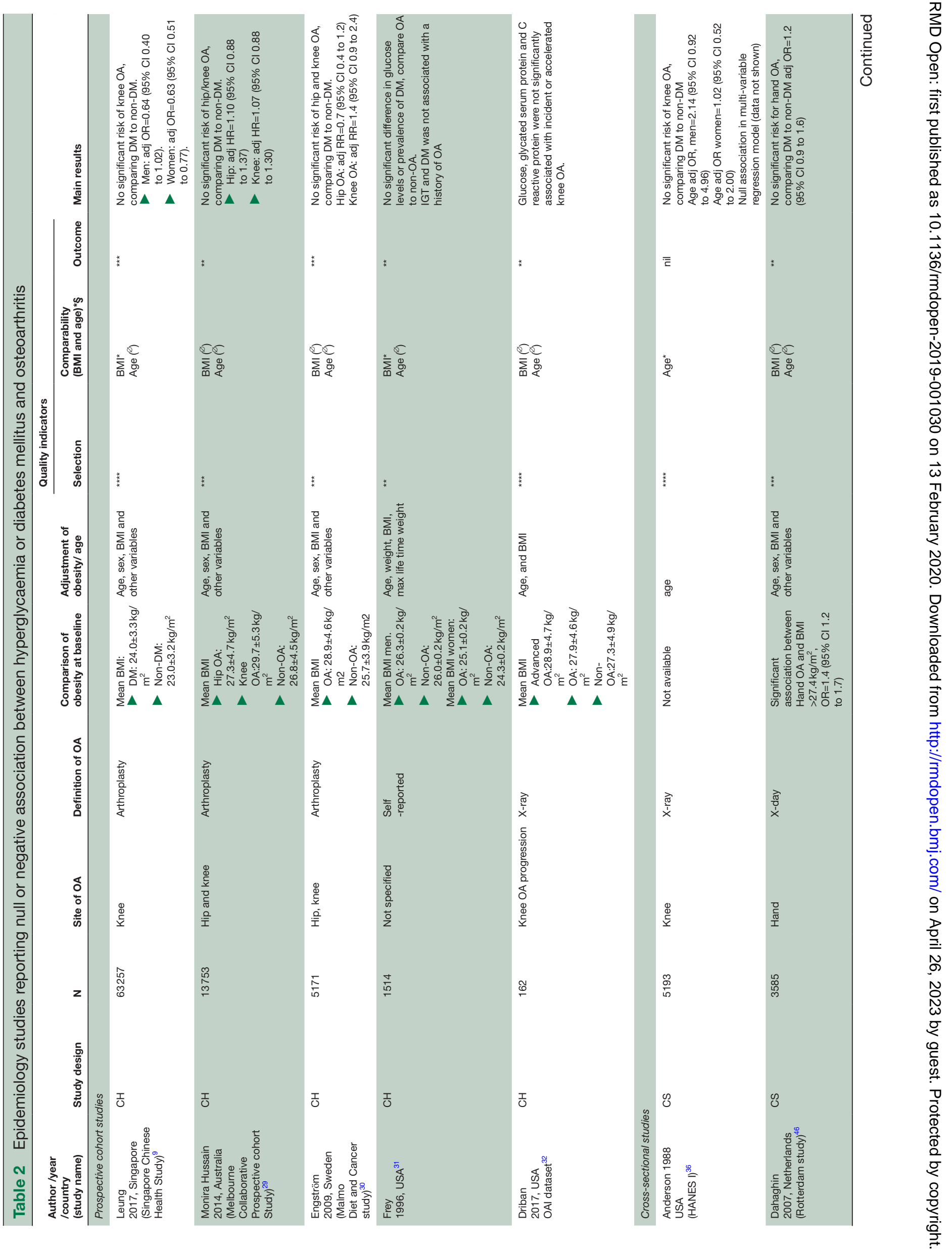




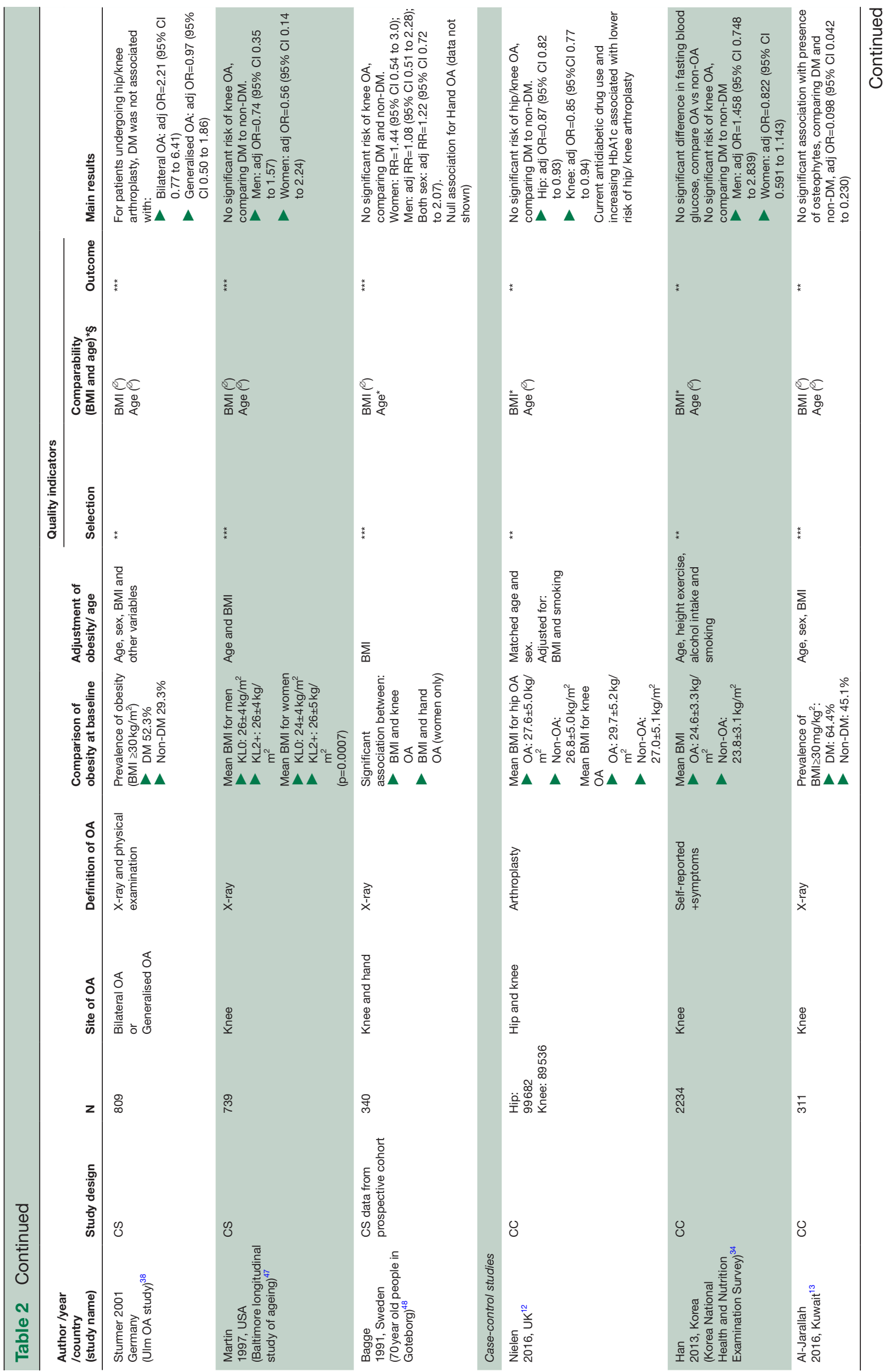




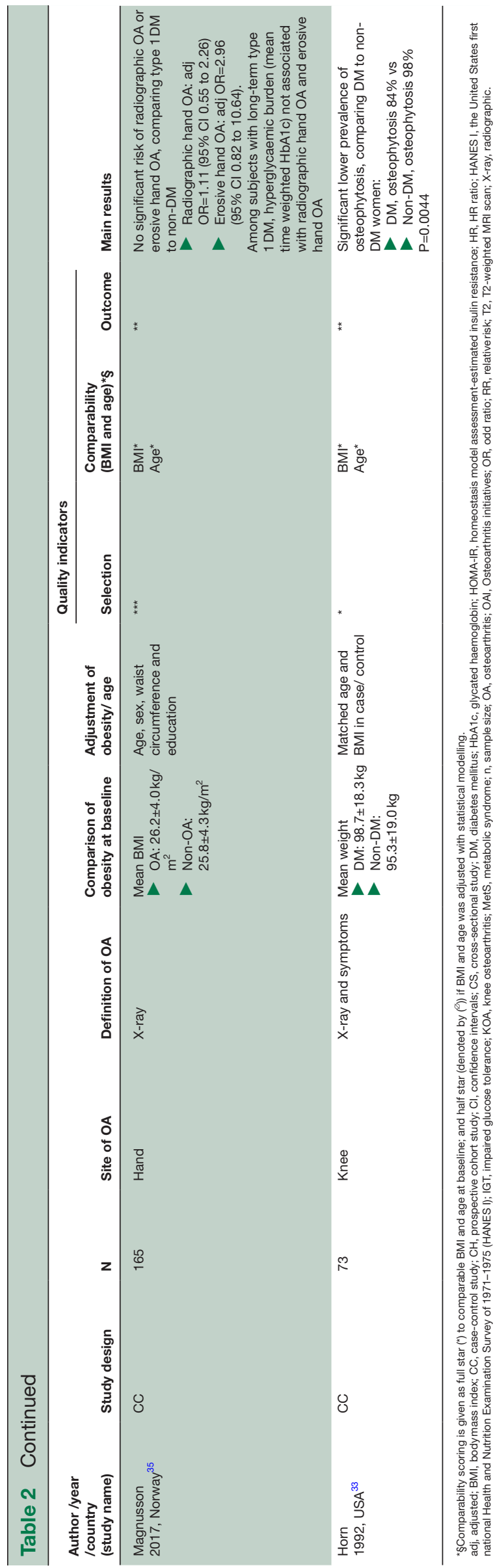

95\% CI 0.49 to 1.38 ) and prospective cohort studies (OR $1.08,95 \%$ CI 0.80 to 1.44 ). Other sensitivity analyses for genders and site of OA did not reveal significant association between OA and DM (table 3). The forest plots of all subgroup analyses are shown in online supplementary figures $2-5$. No publication bias was noted in sensitivity analysis stratified by study design (table 3). Publication bias was noted in the sensitivity analysis for studies in women, but not noted in studies in men (table 3 ).

\section{DISCUSSION}

This systematic review and meta-analysis refute DM as an independent risk factor for OA, when BMI is accounted for. This finding remained consistent regardless of gender and site of $\mathrm{OA}$.

Our findings corroborate with a recent systematic review, ${ }^{14}$ which reported little evidence to suggest that impaired glucose metabolism was a risk factor for OA. This is in contrary to two previous meta-analyses on the same topic. ${ }^{1011}$ There are several strengths of our current findings compared with that of the two previous metaanalyses. First, our updated literature search included several recent and larger studies that reported null/ negative association between DM and OA. ${ }^{12} 29$ Two of these studies were of prospective cohort design, ${ }^{9}{ }^{29}$ while the other was a huge population-based case-control study with cases of hip and knee OA matched to age and gender with population control. ${ }^{12}$ All three recent studies had adjusted for BMI in their final model, one had comparable BMI between those with or without DM for the knee OA model, ${ }^{9}$ while the other two had comparable BMI for cases of OA versus non-OA cases in the hip OA model (but not for knee model). ${ }^{12}{ }^{29}$ Second, we used the best adjusted OR which included BMI, the strongest confounding factor in our meta-analysis. In our qualitative systematic review, we have demonstrated that there were fewer positive studies that had adjusted for BMI. BMI is an established and strong risk factor for both $\mathrm{DM}^{4}$ and $\mathrm{OA}$, particularly for weight-being joints. It is therefore not unexpected that in most of these studies conducted among Western populations, subjects with diabetes or hyperglycemia had much higher BMI, or higher proportion of obesity compared with those without these conditions. ${ }^{67133338}$ For example, in a prospective cohort study from Italy, ${ }^{7}$ the mean baseline BMI in subjects with DM was $27.0 \mathrm{~kg} / \mathrm{m}^{2}$ compared with $24.8 \mathrm{~kg} / \mathrm{m}^{2}$ for subjects without DM. Given the large discrepancy between baseline characteristic, it is challenging to eliminate residual confounding effect of obesity on the association between diabetes and knee OA risk, even after adjusting for BMI with statistical modelling.

The result of our study may be explained by two possibilities. First, DM is truly not a risk factor for OA. Second, when BMI is such a strong risk factor for OA, any weaker risk factors for OA would be masked by the confounding effect of BMI. Therefore, we proposed 


\begin{tabular}{lcccccc} 
Study name & \multicolumn{5}{c}{ Statistics for each study } \\
\cline { 2 - 6 } & $\begin{array}{c}\text { Odds } \\
\text { ratio }\end{array}$ & $\begin{array}{c}\text { Lower } \\
\text { limit }\end{array}$ & $\begin{array}{c}\text { Upper } \\
\text { limit }\end{array}$ & Z-Value & p-Value \\
Cimmino, (both gender), (finger, hip, knee), unadj & 1.106 & 0.570 & 2.146 & 0.297 & 0.766 \\
Engstrom, (men), (knee), unadj & 1.774 & 0.825 & 3.817 & 1.467 & 0.142 \\
Engstrom, (women), (knee), unadj & 2.865 & 1.596 & 5.143 & 3.527 & 0.000 \\
Frey, (men), (all OA sites) adj & 0.721 & 0.455 & 1.142 & -1.394 & 0.163 \\
Frey, (women), (all OA sites) adj & 1.086 & 0.710 & 1.662 & 0.381 & 0.704 \\
Haugen, (both gender), (radiographic hand OA), unadj & 1.538 & 0.990 & 2.390 & 1.917 & 0.055 \\
Maddah, (men), (knee), unadj & 1.155 & 0.225 & 5.934 & 0.173 & 0.863 \\
Maddah, (women), (knee), unadj & 3.971 & 1.062 & 14.850 & 2.050 & 0.040 \\
Monira Hussain, (both gender), (knee), unadj & 1.412 & 1.192 & 1.673 & 3.986 & 0.000 \\
Nielen, (both gender), (knee), unadj & 1.129 & 1.082 & 1.177 & 5.618 & 0.000 \\
Nieves-Plaza, (both gender), (hand and knee), unadj & 2.669 & 1.481 & 4.811 & 3.265 & 0.001 \\
Puenpatom, (both gender), (all OA sites), unadj & 3.512 & 3.006 & 4.104 & 15.812 & 0.000 \\
Rahman, (both gender), (all OA sites), unadj & 1.183 & 1.131 & 1.236 & 7.388 & 0.000 \\
Siviero, (both gender), (shoulder), unadj & 1.715 & 1.090 & 2.700 & 2.331 & 0.020 \\
& 1.556 & 1.283 & 1.887 & 4.488 & 0.000
\end{tabular}

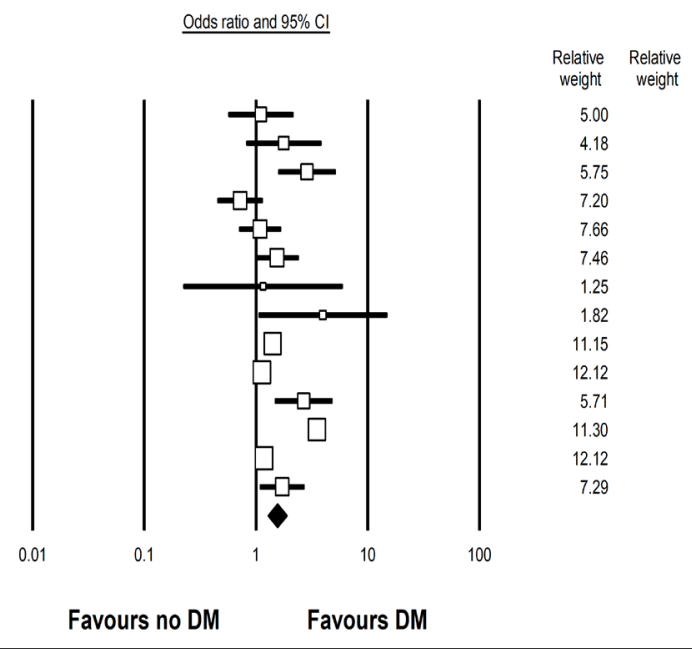

Figure 2 Forest plot on meta-analysis on risk of DM comparing subjects with $\mathrm{OA}$ to those without $\mathrm{OA}(\mathrm{OR} 1.56,95 \% \mathrm{Cl} 1.28$ to 1.89). Legend: Study name were described as first author, gender, OA site, adjusted or unadjusted for BMI. BMI, body mass index; DM, diabetes mellitus; OA, osteoarthritis.

that the true risk estimates attributed by DM independently towards OA may be revealed in populations where BMI is more comparable between comparison groups. Indeed, we have demonstrated that among the seven studies that had comparable baseline BMI between subjects with or without DM, ${ }^{9} 203133-3537$ only one study showed a positive association between DM and $\mathrm{OA},{ }^{20}$ four did not show an association, ${ }^{31} 343537$ while two showed a negative association between DM and OA. ${ }^{933}$ We found no publication bias in this model. Limiting to studies with comparable BMI between groups at baseline may allow the possible biometabolic effect of DM on risk of OA to be revealed more independently from the biomechanical effect of BMI. Of note is that we used an arbitrary BMI of within $1 \mathrm{~kg} /$ $\mathrm{m}^{2}$ difference to define comparability, which may not

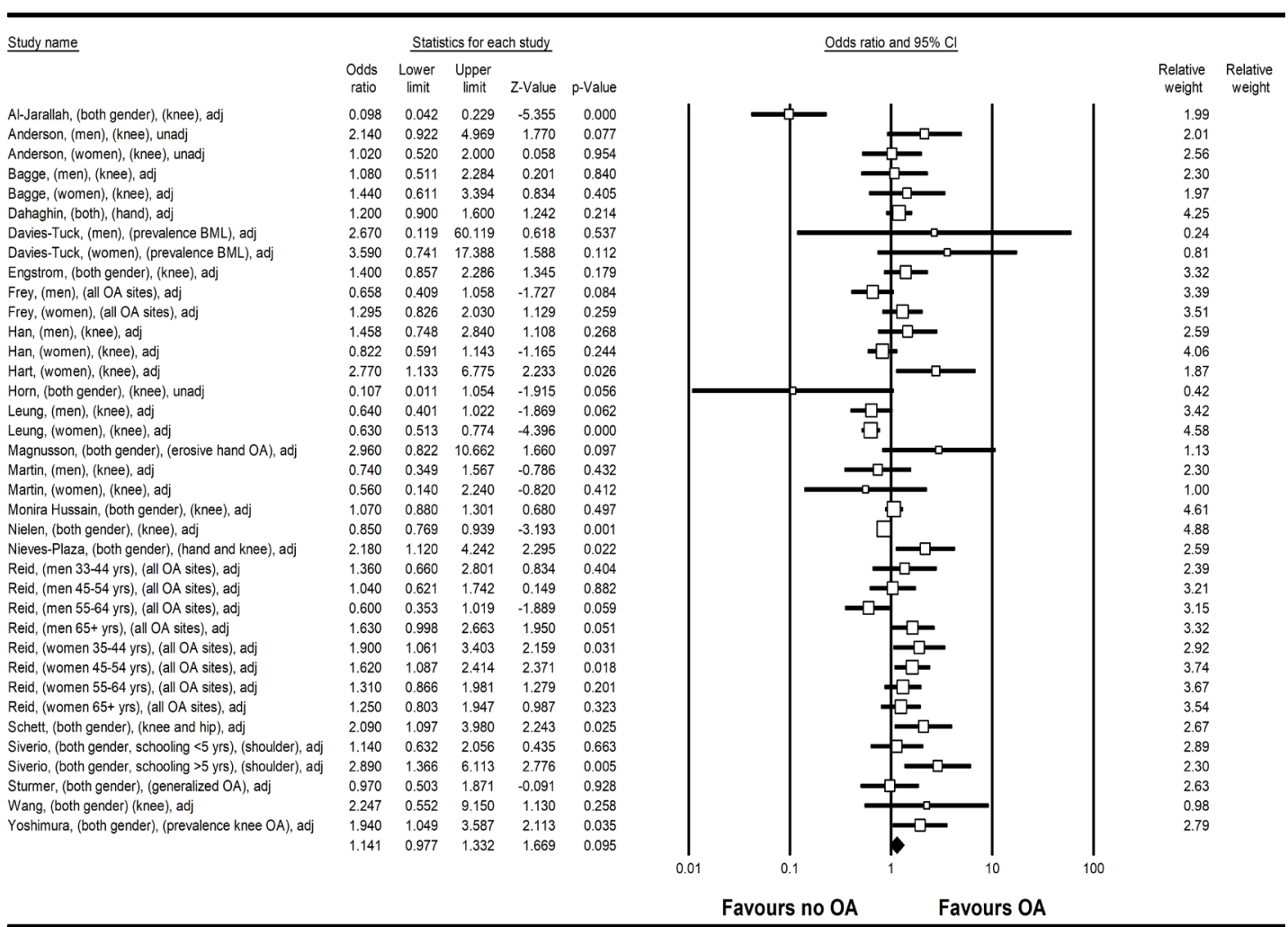

Figure 3 Forest plot on meta-analysis on risk of OA comparing subjects with DM and to those without DM (OR 1.14, 95\% Cl 0.98 to 1.33). Legend: Study name were described as first author, gender, OA site, adjusted or unadjusted for BMI. BMI, body mass index; DM, diabetes mellitus; OA, osteoarthritis. 
Table 3 Summary of risk estimates of the association between diabetes or hyperglycaemia and osteoarthritis

\begin{tabular}{|c|c|c|c|c|c|}
\hline & $\begin{array}{l}\text { No of } \\
\text { studies }\end{array}$ & OR $(95 \% \mathrm{Cl})$, p value & $I^{2}(\%)$ & $\begin{array}{l}\text { P for } \\
\text { heterogeneity }\end{array}$ & $\begin{array}{l}\text { Egger's test for } \\
\text { publication bias ( } p \text { value) }\end{array}$ \\
\hline Overall & 22 & 1.14 (0.91 to 1.03$), p=0.10$ & 74.2 & $<0.01$ & 0.02 \\
\hline \multicolumn{6}{|l|}{ Comparability } \\
\hline $\begin{array}{l}\text { Studies with any adjustment of BMI } \\
28-3134353842132023-26\end{array}$ & 20 & 1.14 (0.97 to 1.34$), p=0.10$ & 74.3 & $<0.01$ & 0.01 \\
\hline $\begin{array}{l}\text { Studies with comparable baseline BMI between } \\
\text { groups }^{903133-35}\end{array}$ & 6 & 0.94 (0.68 to 1.29$), p=0.68$ & 72.6 & $<0.01$ & 0.23 \\
\hline \multicolumn{6}{|l|}{ Study design } \\
\hline Prospective cohort ${ }^{7} 2023$ 29-31 & 7 & 1.08 (0.80 to 1.44$), p=0.63$ & 76.0 & $<0.01$ & 0.14 \\
\hline Case-control $^{8121333-35}$ & 6 & 0.82 (0.49 to 1.38$), p=0.45$ & 85.7 & $<0.01$ & 0.89 \\
\hline Cross-sectional ${ }^{24-26} 28363846-48$ & 9 & 1.29 (1.10 to 1.51$), p<0.01$ & 30.0 & 0.10 & 0.68 \\
\hline \multicolumn{6}{|l|}{ Gender } \\
\hline Men $^{923283134364748}$ & 8 & 1.00 (0.76 to 1.31$), p=0.99$ & 49.4 & 0.03 & 0.22 \\
\hline Women' 2326283134364748 & 9 & 1.24 (0.92 to 1.67$), p=0.16$ & 75.6 & $<0.01$ & $<0.01$ \\
\hline \multicolumn{6}{|l|}{ Site of OA } \\
\hline Hand $\mathrm{OA}^{24313546}$ & 4 & 1.26 (0.77 to 2.05$), p=0.36$ & 69.0 & 0.01 & 0.66 \\
\hline Hip OA ${ }^{1229-31}$ & 4 & 0.90 (0.74 to 1.09$), p=0.27$ & 52.5 & 0.08 & 0.88 \\
\hline Knee $\mathrm{OA}^{912132023242629-313334364748}$ & 15 & 0.97 (0.79 to 1.18$), p=0.75$ & 72.1 & $<0.01$ & 0.27 \\
\hline
\end{tabular}

$\mathrm{BMI}$, body mass index; $\mathrm{Cl}$, confidence intervals; $\mathrm{OA}$, osteoarthritis; OR, odd ratio.

be truly comparable. However, it is a relatively smaller difference compared with the other studies. ${ }^{7}$ In our sensitivity analyses, we asked whether the association between DM and OA may only be applicable to certain subgroups, including women and non-weight bearing joints. The notion of no significant association between DM and OA remained, apart from the subgroup analysis for cross-sectional studies which reached statistical significance.

Several in vitro and in vivo studies have suggested that increased oxidative stress, cytokines and the accumulation of advanced end glycation products (AGE) may link hyperglycaemia to OA. ${ }^{39} 40$ However, BMI was not accounted for in these studies. Even in experimental animal studies, it is difficult to separate the biomechanical effects of obesity from the biometabolic effects of diabetes. ${ }^{5}$ All diabetes animal models, either induced from genetic manipulation or high fat diet, were more obese than the control animals without induced diabetes. ${ }^{5}$ Laiguillon et al reported greater interleukin-6 and prostaglandin E2 expression on stimulation with interleukin-1 $\beta$ comparing human cartilage taken from OA subjects with DM to those without DM. ${ }^{39}$ However, subjects with DM in their study weighed on average $5 \mathrm{~kg}$ higher compared with their non-DM counterparts. Similarly, Zhang et al reported significantly higher levels of AGE in synovial fluid in subjects with DM compared with those without. ${ }^{40}$ Again, subjects with DM had significantly higher BMI than those without DM. It has been postulated that obesity results in an increased state of systemic inflammation with increase in inflammatory cytokine, adipokine and acute phase reactant production ${ }^{41}$ which may link to insulin resistant, and mimic those seen in OA. The implications of our current study that refuted an association of $\mathrm{DM}$ and risk of $\mathrm{OA}$ indicate that $\mathrm{BMI}$ is a strong confounding factor which cannot be ignored in analysis of the relationship between DM and OA. Until further mechanistic causative link is found between DM and $\mathrm{OA}$ on the molecular level, caution should be exercised in interpreting the association between DM and OA from epidemiological studies.

The main limitation of the current study was the moderate to substantial heterogeneity among the studies $\left(\mathrm{I}^{2}=74 \%\right)$. This was likely related to the expected diversity in the characteristics of the population from the various studies. Therefore, the random effect models have been applied. Attempts have been made to mitigate this through sensitivity analyses in various subgroups, although a moderate to substantial degree of heterogeneity was still present. Second, majority of the studies in the analysis were retrospective cross-sectional and case-control design. As community-based prospective cohort studies measure potential exposures before the outcome of interest, they are generally subject to less risk of recall bias and selection bias, although attrition bias may present. ${ }^{42}$ There were higher number of prospective cohort studies in those reported null/negative association between the DM and OA, yet the total number of prospective cohort studies was less than one third. Apart from one study that was specific to type $1 \mathrm{DM},{ }^{35}$ all studies did not specify type of DM. Therefore, the results of this meta-analysis may only be generalisable to a mixed population with type 1 and type $2 \mathrm{DM}$.

\section{CONCLUSION}

In summary, we report the first meta-analysis showing no association between DM and OA. Future research should 
account for the effects of BMI as it is a major confounding factor in the development of OA.

Contributors YYL and CM conceptualised and designed the study. AK, $\mathrm{CM}, \mathrm{CH}$, $\mathrm{LH}$ and YYL acquired the data. AK and YYL performed the data analysis. All authors interpreted the data. AK and YYL drafted the manuscript. All authors critically revised the manuscript and approved the final version of manuscript.

Funding YYL was supported by the National Medical Research Council, Singapore (NMRC/CSAINV/0022/2017).

Competing interests None declared.

Patient consent for publication Not required.

Provenance and peer review Not commissioned; externally peer reviewed.

Data availability statement All data relevant to the study are included in the article or uploaded as supplementary information. Data are available on reasonable request. Request can be made by email to the correspondent author.

Open access This is an open access article distributed in accordance with the Creative Commons Attribution Non Commercial (CC BY-NC 4.0) license, which permits others to distribute, remix, adapt, build upon this work non-commercially, and license their derivative works on different terms, provided the original work is properly cited, appropriate credit is given, any changes made indicated, and the use is non-commercial. See: http://creativecommons.org/licenses/by-nc/4.0/.

ORCID iDs

Andrew Khor http://orcid.org/0000-0002-4675-628X

Ying Ying Leung http://orcid.org/0000-0001-8492-6342

\section{REFERENCES}

1 Vos T, Flaxman AD, Naghavi M, et al. Years lived with disability (YLDs) for 1160 sequelae of 289 diseases and injuries 1990-2010: a systematic analysis for the global burden of disease study 2010 . The Lancet 2012;380:2163-96.

2 Jiang L, Tian W, Wang Y, et al. Body mass index and susceptibility to knee osteoarthritis: a systematic review and meta-analysis. Joint Bone Spine 2012;79:291-7.

3 Leung Y-Y, Allen JC, Noviani M, et al. Association between body mass index and risk of total knee replacement, the Singapore Chinese Health study. Osteoarthritis and Cartilage 2015;23:41-7.

4 Guh DP, Zhang W, Bansback N, et al. The incidence of comorbidities related to obesity and overweight: a systematic review and meta-analysis. BMC Public Health 2009;9:88.

5 King KB, Rosenthal AK. The adverse effects of diabetes on osteoarthritis: update on clinical evidence and molecular mechanisms. Osteoarthritis and Cartilage 2015;23:841-50.

6 Waine H, Nevinny D, Rosenthal J, et al. Association of osteoarthritis and diabetes mellitus. Tufts Folia Med 1961;7:13-19.

7 Schett G, Kleyer A, Perricone C, et al. Diabetes is an independent predictor for severe osteoarthritis: results from a longitudinal cohort study. Diabetes Care 2013;36:403-9.

8 Nieves-Plaza M, Castro-Santana LE, Font YM, et al. Association of hand or knee osteoarthritis with diabetes mellitus in a population of Hispanics from Puerto Rico. J Clin Rheumatol 2013;19:1-6.

9 Leung $Y-Y$, Allen JC, Ang L-W, et al. Diabetes mellitus and the risk of total knee replacement among Chinese in Singapore, the Singapore Chinese Health study. Sci Rep 2017;7:40671.

10 Louati K, Vidal C, Berenbaum F, et al. Association between diabetes mellitus and osteoarthritis: systematic literature review and metaanalysis. RMD Open 2015;1:e000077.

11 Williams MF, London DA, Husni EM, et al. Type 2 diabetes and osteoarthritis: a systematic review and meta-analysis. J Diabetes Complications 2016;30:944-50.

12 Nielen JTH, Emans PJ, van den Bemt B, et al. Association of type 2 diabetes mellitus with self-reported knee pain and clinical knee osteoarthritis: the Maastricht study. Diabetes Metab 2018;44:296-9.

13 Al-Jarallah K, Shehab D, Abdella N, et al. Knee osteoarthritis in type 2 diabetes mellitus: does insulin therapy retard osteophyte formation? Med Princ Pract 2016;25:12-17.

14 Dawson LP, Fairley JL, Papandony MC, et al. Is abnormal glucose tolerance or diabetes a risk factor for knee, hip, or hand osteoarthritis? A systematic review. Semin Arthritis Rheum 2018;48:176-89.

15 Wells G, Shea B, O'Connell D, et al. The Newcastle-Ottawa scale (NOS) for assessing the quality of nonrandomised studies in metaanalyses 2013 .
16 Borenstein M, Hedges LV, Higgins JPT, et al. A basic introduction to fixed-effect and random-effects models for meta-analysis. Res. Synth. Method 2010;1:97-111.

17 Egger M, Smith GD, Schneider M, et al. Bias in meta-analysis detected by a simple, graphical test. BMJ 1997;315:629-34.

18 Duval S, Tweedie R. A Nonparametric "Trim and Fill" Method of Accounting for Publication Bias in Meta-Analysis. Journal of the American Statistical Association 2000;95:89-98.

19 Rahman MM, Kopec JA, Cibere J, et al. The relationship between osteoarthritis and cardiovascular disease in a population health survey: a cross-sectional study. BMJ Open 2013;3:e002624.

20 Yoshimura N, Muraki S, Oka H, et al. Accumulation of metabolic risk factors such as overweight, hypertension, dyslipidaemia, and impaired glucose tolerance raises the risk of occurrence and progression of knee osteoarthritis: a 3-year follow-up of the road study. Osteoarthritis and Cartilage 2012;20:1217-26.

21 Haugen IK, Ramachandran VS, Misra D, et al. Hand osteoarthritis in relation to mortality and incidence of cardiovascular disease: data from the Framingham heart study. Ann Rheum Dis 2015:74:74-81.

22 Karvonen-Gutierrez CA, Sowers MFR, Heeringa SG. Sex dimorphism in the association of cardiometabolic characteristics and osteophytes-defined radiographic knee osteoarthritis among obese and non-obese adults: NHANES III. Osteoarthritis and Cartilage 2012;20:614-21.

23 Davies-Tuck ML, Wang Y, Wluka AE, et al. Increased fasting serum glucose concentration is associated with adverse knee structural changes in adults with no knee symptoms and diabetes. Maturitas 2012;72:373-8.

24 Wang Y, Peng R, Ma R. Epidemiological investigation of osteoarthritis in middle-aged Mongolian and senior residents of the inner Mongolia autonomous region. Iran Red Crescent Med J 2013;15:e8303

25 Siviero P, Tonin P, Maggi S. Functional limitations of upper limbs in older diabetic individuals. The Italian longitudinal study on aging. Aging Clin Exp Res 2009;21:458-62.

26 Hart DJ, Doyle DV, Spector TD. Association between metabolic factors and knee osteoarthritis in women: the Chingford study. $J$ Rheumatol 1995;22:1118-23.

27 Eymard F, Parsons C, Edwards $\mathrm{MH}$, et al. Diabetes is a risk factor for knee osteoarthritis progression. Osteoarthritis and Cartilage 2015;23:851-9.

28 Reid JL, Morton DJ, Wingard DL, et al. Obesity and other cardiovascular disease risk factors and their association with osteoarthritis in southern California American Indians, 2002-2006. Ethn Dis 2010;20:416-22.

29 Monira Hussain S, Wang Y, Cicuttini FM, et al. Incidence of total knee and hip replacement for osteoarthritis in relation to the metabolic syndrome and its components: a prospective cohort study. Semin Arthritis Rheum 2014;43:429-36.

30 Engström G, Gerhardsson de Verdier M, Rollof J, et al. C-Reactive protein, metabolic syndrome and incidence of severe hip and knee osteoarthritis. A population-based cohort study. Osteoarthritis and Cartilage 2009;17:168-73.

31 Frey MI, Barrett-Connor E, Sledge PA, et al. The effect of noninsulin dependent diabetes mellitus on the prevalence of clinical osteoarthritis. A population based study. J Rheumatol 1996;23:716-22

32 Driban JB, Eaton CB, Amin M, et al. Glucose homeostasis influences the risk of incident knee osteoarthritis: data from the osteoarthritis initiative. J Orthop Res 2017;35:2282-7.

33 Horn CA, Bradley JD, Brandt KD, et al. Impairment of osteophyte formation in hyperglycemic patients with type II diabetes mellitus and knee osteoarthritis. Arthritis \& Rheumatism 1992;35:336-42.

34 Han CD, Yang IH, Lee WS, et al. Correlation between metabolic syndrome and knee osteoarthritis: data from the Korean National health and nutrition examination survey (KNHANES). BMC Public Health 2013;13:603.10.1186/1471-2458-13-603

35 Magnusson K, Bech Holte K, Juel NG, et al. Long term type 1 diabetes is associated with hand pain, disability and stiffness but not with structural hand osteoarthritis features - the Dialong hand study. PLoS One 2017;12:e0177118.

36 Anderson JJ, Felson DT. Factors associated with osteoarthritis of the knee in the first National health and nutrition examination survey (Hanes I). Evidence for an association with overweight, race, and physical demands of work. Am J Epidemiol 1988;128:179-89.

37 Shin D. Association between metabolic syndrome, radiographic knee osteoarthritis, and intensity of knee pain: results of a national survey. J Clin Endocrinol Metab 2014;99:3177-83.10.1210/jc.20141043 
38 Sturmer T, Brenner H, Brenner RE, et al. Non-Insulin dependent diabetes mellitus (NIDDM) and patterns of osteoarthritis. The UIm osteoarthritis study. Scand J Rheumatol 2001;30:169-71.

39 Laiguillon M-C, Courties A, Houard X, et al. Characterization of diabetic osteoarthritic cartilage and role of high glucose environment on chondrocyte activation: toward pathophysiological delineation of diabetes mellitus-related osteoarthritis. Osteoarthritis Cartilage 2015;23:1513-22.

40 Zhang W, Randell EW, Sun G, et al. Hyperglycemia-related advanced glycation end-products is associated with the altered phosphatidylcholine metabolism in osteoarthritis patients with diabetes. PLoS One 2017;12:e0184105.

41 Gregor MF, Hotamisligil GS. Inflammatory mechanisms in obesity. Annu Rev Immunol 2011;29:415-45.

42 Mann CJ. Observational research methods. research design II: cohort, cross sectional, and case-control studies. Emerg Med J 2003;20:54-60.
43 Puenpatom RA, Victor TW. Increased prevalence of metabolic syndrome in individuals with osteoarthritis: an analysis of NHANES III data. Postgrad Med 2009;121:9-20.

44 Cimmino MA, Cutolo M. Plasma glucose concentration in symptomatic osteoarthritis: a clinical and epidemiological survey. Clin Exp Rheumatol 1990;8:251-7.

45 Maddah S, Mahdizadeh J. Association of metabolic syndrome and its components with knee osteoarthritis. Acta Med Iran 2015;53:743-8.

46 Dahaghin S, Bierma-Zeinstra SMA, Koes BW, et al. Do metabolic factors add to the effect of overweight on hand osteoarthritis? the Rotterdam study. Ann Rheum Dis 2007;66:916-20.

47 Martin K, Lethbridge-Cejku M, Muller DC, et al. Metabolic correlates of obesity and radiographic features of knee osteoarthritis: data from the Baltimore longitudinal study of aging. J Rheumatol 1997;24:702-7.

48 Bagge E, Bjelle A, Eden S, et al. Factors associated with radiographic osteoarthritis: results from the population study 70-year-old people in Goteborg. J Rheumatol 1991;18:1218-22. 